\title{
Objetivación del límite de una función: ¿pensamiento empírico o pensamiento teórico? ${ }^{1}$
}

\author{
Objectification of the limit of a function: empirical \\ or theoretical thinking thought?
}

Objetivação do limite de uma função: o pensamento

pensamento empírica ou teórica?

Recibido: mayo de 2013

Aceptado: agosto de 2013
Claudia Patricia Quintero Quintero²

Diana Victoria Jaramillo Quiceno ${ }^{3}$

\section{Resumen}

Presentamos un avance del proyecto de tesis de doctorado que estamos realizando en el marco del Doctorado en Educación, Línea de Educación Matemática, de la Universidad de Antioquia. Este estudio tiene como propósito analizar la objetivación del concepto de límite de una función, de alumnas de grado once, a través del desarrollo de su pensamiento teórico. La perspectiva histórico-cultural de la educación sirve de fundamentación teórica en esta investigación, en especial la Teoría de la Actividad. El camino metodológico a seguir es de orden cualitativo, desde un paradigma crítico-dialéctico, y una investigación participante. El trabajo de campo se realizará en una institución escolar pública de Medellín.

Palabras clave: Investigación e innovación en Educación Matemática; Marcos teóricos; Teorías de aprendizaje; Situado sociocultural; Perspectiva histórico-cultural de la educación matemática; Lógica dialéctica; Teoría de la Actividad; Actividades Orientadoras de Enseñanza.

\begin{abstract}
We present a preview of the doctoral thesis project we are doing in the context of the Doctorate in Education Online Mathematics Education at the University of Antioquia. This study aims to analyze the objectification of the concept of limit of a function, of eleventh grade students, through the development of his theoretical thinking. The cultural-historical perspective of education serves as theoretical basis of this research, especially the Activity Theory. The methodological approach to follow is of a qualitative, from a critical-dialectical paradigm, and research participant. The fieldwork was conducted in a public educational institution of Medellin.
\end{abstract}

Keywords: Research and Innovation in Mathematics Education, Theoretical frameworks, learning theories, Situated sociocultural, historical and cultural perspective of mathematics education; Logic dialectical Activity Theory; Guiding Teaching Activities.

1 Artículo de Investigación.

2 Universidad de Antioquia, Matemáticas, “Educación y Sociedad-MES”. Medellin, Colombia. Contacto: clauquinte22@yahoo.com

3 Universidad de Antioquia, Matemáticas, Educación y Sociedad-MES”. Medellin, Colombia. Contacto:diana_jaramillo@hotmail.com 


\section{Resumo}

Nós apresentamos uma prévia do projeto de tese de doutorado que estamos fazendo no contexto do Doutorado em Educação Online Educação Matemática na Universidade de Antioquia. Este estudo tem como objetivo analisar a objetivação do conceito de limite de uma função, de estudantes décimo primeiro grau, através do desenvolvimento de seu pensamento teórico. A perspectiva histórico-cultural da educação serve como base teórica desta pesquisa, especialmente a Teoria da Atividade. A abordagem metodológica a seguir é de uma pesquisa qualitativa, a partir de um paradigma crítico-dialético, e participante da pesquisa. O trabalho de campo foi realizado em uma instituição de ensino público de Medellín.

Palavras-chave: Pesquisa e Inovação em Educação Matemática, referenciais teóricos, as teorias de aprendizagem, Situado perspectiva sócio-cultural, histórico e cultural da educação matemática; Logic Teoria da Atividade dialética; Ensino Orientadores Atividades.

\section{Planteamiento del problema}

La definición axiomática de límite ha llegado a nuestras aulas con el propósito de fundamentar la matemática escolar en el nivel medio, conllevando a la organización de los contenidos bajo la influencia de la estructura formal del Análisis Matemático, como lo anotan Molfino y Buendía (2010). Tal fundamentación rigurosa ha propendido por un dominio algorítmico de límite, mas no por una conceptualización real del mismo, desencadenado, así, una problemática para la educación matemática. Problemática que se hace evidente en las dificultades a las que se enfrentan los alumnos en el aprendizaje de dicho concepto ya las que se enfrentan los maestros para su enseñanza.

Según Davidov (1998), la escuela debe practicar una enseñanza desarrollante. Es decir, una enseñanza que forme en los alumnos un pensamiento teórico. Esto es, un pensamiento que posibilite a los alumnos pensar teóricamente: comprender y dominar el proceso de origen y desarrollo de las cosas por medio del análisis de las condiciones de las mismas. El mismo autor afirma que en la escuela se practica una enseñanza que fomenta en los alumnos un pensamiento empírico; este pensamiento dificulta el acercamiento teórico de los alumnos al conocimiento e influye poco en el desarrollo de sus capacidades intelectuales.
Así, desde nuestra práctica pedagógica, nos preguntamos si estaremos posibilitando el desarrollo de la capacidad intelectual de los alumnos, de forma tal que les posibilite adquirir, teóricamente-en el sentido de Davidov (1988) - los conocimientos matemáticos propuestos en el currículo escolar. En particular, nos hacemos esta indagación sobre el concepto de límite de una función, dada la importancia que se le da a la enseñanza de este concepto en el grado once (en el ámbito colombiano) y dada la dificultad de su aprendizaje por parte de los alumnos.

De acuerdo con lo anteriormente expuesto, nos surge la siguiente pregunta de investigación: ¿Cómo es la objetivación del concepto de límite de una función, de alumnas de grado once, a través del desarrollo de su pensamiento teórico? Consecuentemente, es el propósito de este estudio analizar la objetivación del concepto de límite de una función, de alumnas de grado once, a través del desarrollo de su pensamiento teórico.

\section{Marco teórico}

Nuestras comprensiones teóricas en este estudio, desde los aspectos epistemológicos, ontológicos y gnoseológicos, están inmersas en la perspectiva histórico-cultural de la educación. 
Así, a continuación, explicitaremos algunas de estas comprensiones.

Objetivación: Desde nuestra perspectiva gnoseológica, el sujeto es reconocido como un ser en la cultura. Es decir, la cultura es transformada por el sujeto y, a su vez, el sujeto es transformado por la cultura. Una transformación derivada de un proceso de constitución de "ser con los otros" (Radford, 2004).De esta manera, la cultura posibilita la forma en que el sujeto interpreta el mundo y, por ende, la forma en que produce un conocimiento.

Entendemos esa forma de producir un conocimiento como la objetivación, en el sentido de Radford (2006, 2008). Objetivación asumida como un proceso social donde el conocimiento surge desde la dialéctica individuo/grupo, cuando el sujeto toma conciencia progresiva (Radford, 2006, p. 116) de la esencia del objeto, dotándolo de sentido. Pero, además, el conocimiento y las formas como el sujeto accede a dicho conocimiento, hacen parte de lo que el sujeto es, es decir, de su subjetividad.

Límite de una función. El concepto de límite se comienza a gestar en el siglo XVII, en un momento socio-histórico en el que, según Aleksandrov (1976), se requería transformar el estudio matemático de las leyes del movimiento, ya que se comprendió que el estado de inmovilidad es inexistente en la naturaleza y que, por lo tanto, el cambio es incesante.

De esta manera, según Caraça (1984), surgió el problema del movimiento, el cual consistió esencialmente en la imposibilidad de hallar la posición de un móvil en determinado instante en un punto de su trayectoria, ya que entre dos puntos, por más cercanos que estén, existen infinidad de puntos. Tal problema exigió la creación de un nuevo instrumento matemático: el concepto de infinitésimo. Concepto entendido como la variable representativa de un conjunto de valores sucesivos; valores tan próximos a cero cuanto se desee (Caraça, 1984).

La aplicación del infinitésimo, y su posterior desarrollo, posibilitó la creación del concepto de límite de una sucesión numerable. Este concepto es definido por Caraça (1984, p.231), así2:

Se dice que la sucesión numerable an tiene por límite el número $\mathrm{L}$, cuando $\mathrm{n}$ tiende a infinito.

De esta forma, se observa que L es para la sucesión an, el resultado de la interdependencia de sus términos. En esa dirección, coincidimos con Aleksandrov (1976), en que el concepto de límite permite estudiar dialécticamente cierto estado de un fenómeno en interdependencia con los estados vecinos del mismo.

Si los fenómenos estudiados pueden ser representados por funciones de variable real, entonces el instrumento es el límite de una función real. Este límite es definido por Caraça (1984, p.295)como sigue:

Se dice que $\mathrm{y}(\mathrm{x})$ tiene por límite el número $\mathrm{L}$ cuando $\mathrm{x}$ tiende hacia a, o que $\mathrm{y}(\mathrm{x})$ tiende hacia $\mathrm{L}$ cuando $\mathrm{x}$ tiende hacia a.

Pensamiento empírico. Según Davidov (1988), este tipo de pensamiento no posibilita el desarrollo psíquico del alumno debido a su tipo de generalización y abstracción.

En cuanto a la generalización empírica-propia del pensamiento empírico- puede entenderse, según Davidov (1998), como el movimiento que muestra las características comunes de un objeto o fenómeno en relación a la clase de objetos de la que hace parte. Es decir, consiste en hallar algunas propiedades que se repiten en dichos objetos, o sea, sus invariantes. De acuerdo a este autor:

En la literatura psicodidáctica y sobre métodos de enseñanza, la generalización [empírica] se caracteriza como la vía fundamental para la formación de conceptos en los escolares.

La formación, en los niños, de las generalizaciones [empíricas] conceptuales se considera una de las finalidades principales de la enseñanza escolar. (Davidov, 1988, p.101).

\footnotetext{
2 Las citas de Caraça (1984) y de Moura (2010) son traducidas del portugués al español por las autoras de esta comunicación.
} 
De acuerdo con Davidov (1988), la generalización empírica se encuentra estrechamente relacionada con el proceso de abstracción, ya que para identificar una cualidad común de un objeto se requiere hacer una separación de otras de sus cualidades. Así, en esta perspectiva, lo general se considera sólo como lo igual o semejante en el grupo de objetos o fenómeno. Este tipo de consideraciones coincide, según Davidov (1988), con la interpretación de la lógica formal, lo cual lleva a formar conceptos empíricos en los alumnos, mas no develan la particularidad de los conceptos científicos.

Pensamiento teórico. Davidov (1988) expone que, contrariamente al pensamiento empírico, el pensamiento teórico posibilita al sujeto comprender la esencia del objeto estudiado, mediante la elaboración de los datos observados mediante el método dialéctico. Esto es, hallar las conexiones internas del sistema de los datos contemplados, sus contradicciones y singularidades, como parte de un todo integrado. De esta forma, como lo expresa el mismo autor, en el pensamiento teórico el concepto surge como un modo de actividad psíquica del sujeto que le posibilita la reproducción mental del objeto.

Las principales características del pensamiento teórico, según Moura (2010), son: la transformación del saber en teoría desarrollada mediante deducción y explicación; la elaboración por medio del análisis del papel y de la función de cierta relación entre las cosas en el interior de un sistema; la expresión por diferentes sistemas semióticos; la fundamentación en la transformación de los objetos; la presentación de una forma universal que caracteriza simultáneamente un representante de una clase y un objeto particular; la relación entre lo general y lo particular; y la representación de la relación entre las propiedades del objeto y sus conexiones internas (p.75).

Concordando con Davidov (1988), es nuestra apuesta plantear la necesidad de transformar nuestras prácticas de enseñanza a otras que generen, a su vez, una transformación del pensamiento de nuestros alumnos. Un pensamiento que trasgreda la generalización empírica (producto del simple establecimiento de relaciones mediante la sola percepción), hacia un pensamiento que posibilite un análisis dialéctico del objeto o fenómeno a ser aprendido; esto es, hacia un pensamiento teórico.

Actividad Orientadora de Enseñanza. La Actividad Orientadora de Enseñanza, desde Moura (2010), se encuentra en el marco de la Teoría de la Actividad.

La Teoría de la Actividad es concebida por Leontiev (referenciado por Davidov, 1988) como:

Una secuencia dialécticamente interconectada de acciones mediatizadas a través de las cuales los individuos se relacionan no solamente con el mundo de los objetos sino que también con otros individuos, adquiriendo, en el curso de ese proceso, la experiencia humana.

Así, la Teoría de la Actividad podría, además, entenderse como las formas de organización al interior de una cultura, orientadas a un fin determinado. Moura (1998), partiendo de esta teoría, propone las Actividades Orientadoras de Enseñanza como una forma de organización de la actividad de enseñanza. Dichas Actividades Orientadoras de Enseñanza sugieren pensar, planear y desarrollar los encuentros en el aula de clase, de tal manera que se generen interacciones que posibiliten retomar el conocimiento matemático socialmente construido. Esas Actividades son una alternativa para potenciar en el alumno el desarrollo del pensamiento teórico.

\section{Metodología}

Como es nuestra intencionalidad estudiar la objetivación del concepto de límite de una función, de alumnas de grado once, a través del desarrollo de su pensamiento teórico, la producción de registros y de datos será con tres alumnas de dicho grado de una institución femenina, de carácter oficial de la ciudad de Medellín. Desarrollaremos, al interior del aula, unas Actividades Orientadoras de Enseñanza (Moura, 1998).En las clases haremos nuestros registros a través de: videograbaciones; fotografías; entrevistas a las alumnas; diario reflexivo de investigación. Consideraremos también algunas producciones escritas de las alumnas. El análisis de los datos lo realizaremos mediante el estudio de casos (Yin, 1984) y adoptando una triangulación 
entre los datos, nuestras miradas como investigadoras y el marco teórico.

El estudio lo realizaremos desde del paradigma cualitativo (Denzin, Guba y Lincoln, 1994), bajo un enfoque crítico-dialéctico (Sánchez, 1998), por medio de una investigación participante. Utilizaremos esta metodología dado el interés, a partir de nuestra asunciones epistemológicas, de comunicar y de interpretar una realidad particular de un sujeto. Es este sujeto - alumna- en su contexto escolar, el que nosotras estudiaremos por medio de una participación activa en el aula.

\section{Referencias}

Aleksandrov A. D. (1976). Límites. En: Aleksandrov A.D et al. La Matemática: su contenido, métodos y significado. Versión española de Manuel López Rodríguez. Madrid: Alianza Editorial. p.108-117.

Caraça, B.J. (1984). Conceitos fundamentais da matemática. Lisboa: Livraria Sà Da Costa Editora.

Davídov V. (1988). La enseñanza escolar y el desarrollo psíquico. Investigación psicológica teórica y experimental. Moscú: Progreso.

Denzin, N, Guba, E. y Lincoln, Y. (1994). Competing paradigms in qualitative research. Handbook of qualitative research. Park, CA: SAGE Publications.

Molfino, V. y Buendía G. (2010).El límite de funciones en la escuela: un análisis de su institucionalización. Rev. electrón. investig. educ. cienc. [online] vol.5, n.1, pp. 27-41. ISSN 1850-6666.Extraído el 9 de Marzo de 2013 de: http://www.scielo.org.ar/scielo.php?pi$\mathrm{d}=\mathrm{S} 1850-66662010000100003 \& \mathrm{script}=\mathrm{s}-$ ci_arttext

Moura, M. O. (1998). A atividade de Ensino como Ação Formadora. In: Castro, Amélia

Domingues e CARVALHO, Ana Maria Pessoa de (org.) Ensinar a ensinar. São Paulo: Pioneira Thomson Learning Ltda. 143-162.

Moura, M. O. (2010 Comp). A atividade pedagógica nateoria Histórico- Cultural. Brasilia: Liber libro.

Radford, L. (2006). Elementos de una teoría cultural de objetivación. Revista latinoamericana de Investigación en Matemática educativa. Número especial, p. 103-129.

Radford, L. (2008). The ethics of beingand knowing: Towards a cultural theory of Learning. En: L. Radford, G. Schubring, \&F.eeger (comps.). Semiotics in mathematics education: Epistemology, history, classroom, andculture (pp. 215-234). Rotterdam: SensePublishers. 